\title{
GLACIAL CYCLES AND ICE-SHEET MODELLING
}

\author{
J . OER LEM A N S
}

Institute for Meteorology and Oceanography, University of Utrecht, Princetonplein 5, Utrecht, The Netherlands

\begin{abstract}
An attempt is made to simulate the Pleistocene glacial cycles with a numerical model of the Northern Hemisphere ice sheets. This model treats the vertically-integrated ice flow along a meridian, including computation of bedrock adjustment and temperature distribution in the ice. Basal melt water is traced and controls ice-mass discharge.

The model produces asymmetric glacial cycles, even when it is not forced. Model parameters can be chosen such that cycles with a duration of about 100000 yr occur. Due to the production of basal melt water and bedrock sinking, deglaciations are very rapid.

The occurrence of glacial cycles in the model is a stable feature, but the phase of the cycles is very sensitive to the model parameters. The main conclusion is that ice-sheet dynamics may provide an explanation for the Pleistocene glacial cycles. However, the 'predictability' of the ice-volume record appears to be small.
\end{abstract}

\section{Introduction}

Explaining the quaternary glacial cycles has become one of the major problems in palaeoclimatology. In particular, the apparent $100000 \mathrm{yr}$ power in spectra of the global ice volume record (Hays et al., 1976) is not understood. Most workers agree on the point that the orbital insolation variations at least have a steering effect on the growth and decay of the Northern Hemisphere ice sheets, but it has also been claimed that internal (more or less free) oscillations play the dominant role. Anyway, it has become clear that nonlinearities in the climate system must be responsible for the amplification of the $100000 \mathrm{yr}$ insolation signal (e.g. Birchfield and Weertman, 1978), or, alternatively, internally generate quasi-periodic behaviour on that time scale. Preliminary results of a Northern Hemisphere ice sheet model exhibiting such behaviour were recently reported by the author (Oerlemans, 1980a). It appeared that the interaction of ice-sheet growth and (lagged) bedrock depression may create glacial cycles in the model with a period of about $100000 \mathrm{yr}$.

In this paper experiments with a refined version of this ice-sheet model will be discussed in some detail. The modification consists of computation of the temperature field within the ice sheet, and, if basal melting occurs, dealing with the effect of basal sliding. Before turning to a description of the model, we will first discuss why the Northern Hemisphere ice sheets are likely to play such an important and active role in the establishment of the regular sequence of cold and warm climates during the quaternary epoch.

There have been several approaches in 'modelling the ice ages'. The first models related to this aim were the so-called energy balance climate models, which have become popular through the work of Budyko (1969) and Sellers (1969). With these models, the 
importance of the ice-albedo feedback in increasing the sensitivity of the earth's climate for insolation variations could be investigated. More sophisticated models have been constructed to study, among other things, the effect of different formulations of the heat transport (e.g. Gal-Chen and Schneider, 1976; Lindzen and Farrell, 1977), the potential importance of variations in cloud height (e.g. Schneider, 1972) and cloudiness (Van den Dool, 1980), the possible role of zonal asymmetry (Hartmann and Short, 1979; Oerlemans, 1980b) and the modification of the albedo feedback by the action of the yearly cycle (e.g. North and Coakley, 1979). Recently, a number of studies have been published in which parameterizations employed in energy balance climate models are tested both against observational data (e.g. Warren and Schneider, 1979) and general circulation models (e.g. Coakley and Wielicki, 1979). These studies have cast some doubt on the performance of energy balance climate models under forcing conditions that differ from those to which the models are tuned.

Practically all energy balance climate models have at least two points in common. First, they fail to produce ice ages for realistic changes in the insolation regime. Second, they treat continental ice sheets as if they were pancakes of ice. The latter implies that ice sheets only affect the climate through the ice-albedo feedback - there is no difference between an ice sheet and a snow field in amplifying climate sensitivity. Although Bodvarsson (1955) and Weertman (1961) already showed the extreme importance of the feedback between the ice accumulation rate over an ice sheet and its surface elevation, it has only recently been recognized by climatologists as a potentially very effective mechanism in creating cold climates. Coupling of a simple ice-sheet model to an energy balance climate model was first reported in a note by Pollard (1978), and discussed in more detail by the author (Oerlemans, 1980c) and Pollard et al. (1980). In these studies it is clearly demonstrated that the ice accumulation rate - surface elevation feedback is more important than the ice-albedo feedback. One should thus conclude that 'modelling the ice ages' cannot be done without treating in some detail the basic dynamics of large ice sheets. Moreover, it may ultimately turn out that a Northern Hemisphere ice sheet model on its own is capable of reproducing the quaternary glacial cycles, when directly forced by insolation variations. The first study in this spirit was carried out by Weertman (1976), who showed that the orbital insolation variations may indeed create ice sheets of ice-age size through the feedback between ice-accumulation rate and surface elevation mentioned above. In this interpretation, the Northern Hemisphere ice sheets determine the climatic regime rather than reversed.

Another way to explain the glacial cycles involves free oscillations. Examples are studies by Källen et al. (1979), Ghil and LeTreut (1981), Saltzman et al. (1981) and Sergin (1979). The latter uses a fairly complete model of the climate system. Although these models all exhibit periodic behaviour in some parts of the parameter space involved, it appears that none of them is capable of yielding periods as large as $100000 \mathrm{yr}$. Similar studies include stochastic forcing. It is well-known that the behaviour of nonlinear deterministic systems may change drastically when a stochastic forcing is added. Random diffusion between components of a dynamical system may for example lead to oscillations (e.g. Howard, 1979). Studies essentially based on this concept are those by Nicolis 
and Nicolis (1981) and Sutera (1981). Although these models are as yet far from physical reality, they show that in principle low-frequency oscillations may occur as a result of relatively weak random forcing.

Nonlinear analysis of dynamical systems is in rapid progress and many systems are now known (in particular in biology and ecology) that exhibit relaxation oscillations which look similar to the oscillations occurring in $0^{18} / \mathrm{O}^{16}$ records from deep-sea cores (which measure global ice volume, see Figure 1). Due to the wide parameter ranges that can be used in global climate models (because the strength of most feedback loops is very poorly known), it should not be too difficult to construct a nonlinear few-component system that produces relaxation oscillations with a period of about $100000 \mathrm{yr}$. However, one should be careful with this type of approach, because it is a matter of picking the right dynamical system rather than using physical laws simplified by appropriate scaling. Few-component systems should be validated against, or better, be derived from more sophisticated models before they are used extensively.

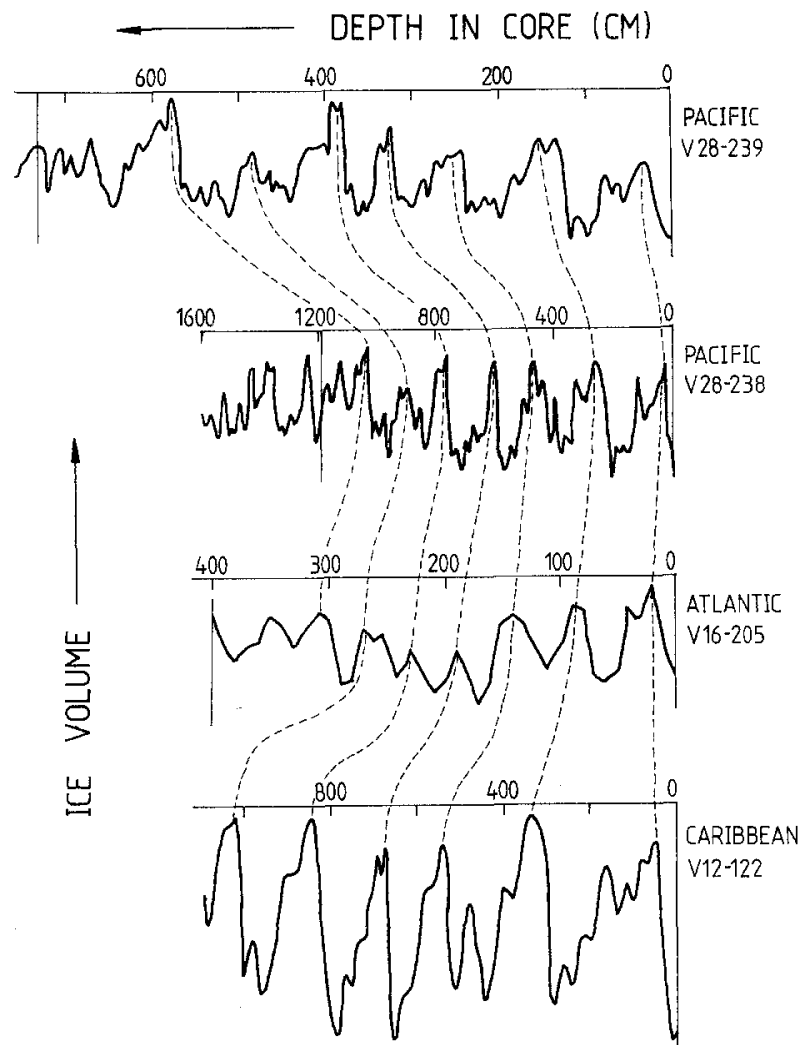

Fig. 1. $O^{18} / O^{16}$ records from deep-sea cores, reflecting variations in the global ice volume. The Brunhes-Matuyama magnetic reversal (about $700000 \mathrm{yr}$ ago) is indicated by thin vertical lines. Dashed lines connect points that are thought to be in phase; the mean spacing is about $100000 \mathrm{yr}$. Refs.: Imbrie et al. (1973), Shackleton and Opdyke (1973, 1976). 
The present study was undertaken in this spirit. Numerical experiments are discussed in which a fairly complete model of the Northern Hemisphere ice sheets is used. We will see that with a model having a large number of degrees of freedom, fairly regular cycles in ice volume can be simulated, even in the absence of forcing. Characteristics of 'observed' ice-volume records are well reproduced, but the results are very sensitive to small variations in the model parameters. This may be due to model deficiencies, but it is more likely that the evolution of the climatic state during the Pleistocene was just one out of many possible realizations.

\section{Model description}

The ice-sheet model to be used is based on the one described in Oerlemans (1981). Recently, Birchfield et al. (1981) employed a similar model. Only vertically-integrated ice flow is computed according to a flow law of the 'Glen-type', in which the vertical mean ice velocity is proportional to the basal shear stress. The model thus comes somewhere between the perfect plasticity models (e.g. Weertman, 1976; Birchfield, 1978) and the models in which the variation of ice velocity with depth is computed explicitly (e.g. Jenssen, 1977). The latter class of models puts too heavy demands on computational resources, at least for palaeoclimatic studies we have in mind.

The geometry of the model, sketched in Figure 2, treats the Northern Hemisphere ice sheets in a very schematic way. Only one sheet is considered. We may imagine that this is the Laurentide ice sheet, which is known to be the most important one, at least when ice volume is concerned. Many objections can be made, however, against the idea of a passive Eurasian ice sheet just following the American one. Anyway, we consider only one model ice sheet, whose evolution is computed along a meridian (north-south, defined here as the $x$-axis).

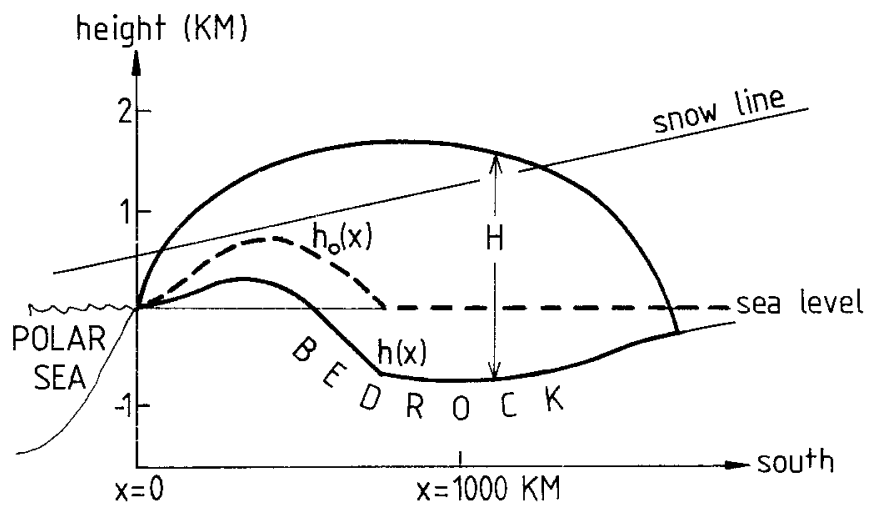

Fig. 2. Schematic picture of the Northern Hemisphere ice-sheet model. Beyond the snow line the ice accumulation rate is positive. The northern edge of the continent is at $73^{\circ} \mathrm{N}$ latitude. The dashed line $\left(h_{0}\right)$ shows the equilibrium bedrock profile when ice load is absent. 
Ice flow is modelled by the following equations

$$
\begin{aligned}
& \frac{\partial H}{\partial t}=\frac{\partial}{\partial x}\left[D \frac{\partial(H+h)}{\partial x}\right]+M \\
& D=B\left(\theta_{0}, W\right) H^{m+1}\left|\frac{\partial(H+h)}{\partial x}\right| m^{-1} \\
& \frac{\partial h}{\partial t}=-\left(H / 3+h-h_{0}\right) / t^{*}
\end{aligned}
$$

In (1)-(3), $H$ is ice thickness, $h$ bedrock elevation, $h_{0}$ bedrock elevation in the undisturbed case (no ice load), $M$ the mass balance or ice accumulation rate. $D$ can be interpreted as a diffusitivity for ice mass. It depends on the flow parameters $B$ and $m$.

Equation (1) describes the conservation of ice mass. The expression in square brackets is the vertically-integrated mass flow (the ice is assumed to be incompressible). In this study $m$ is kept constant at a value of 2.5 , which gives a realistic ice-sheet profile. The flow constant $B$ depends on temperature and the amount of basal water beneath the ice. We turn to this in a moment.

Equation (3) treats the reaction of the bedrock to the ice load. It has been assumed that the density of the upper mantle is about three times that of ice. The constant $t^{*}$ is a time scale, indicating how fast the system returns to isostatic equilibrium $\left(h=h_{0}-H / 3\right)$. Equation (3) does not take into account the flexural rigidity of the lithosphere. This may be a shortcoming for a typical scale of the ice load less than $100 \mathrm{~km}$ (e.g. Turcotte, 1979), i.e. near the edge of an ice sheet. A few experiments were carried out with an equation for $\partial h / \partial t$ that took into account lithospheric rigidity. It appeared to give small deviations from Equation (3), in fact smaller than $75 \mathrm{~m}$, and this has a negligible effect on the evolution of the model ice sheet. If some kind of grounding line dynamics is included (modelling of the ice sheet-ice shelf junction), such deviations near the ice-sheet edge may be important (see Pollard, 1982).

Recently, Pollard (1982) noted that the stability of ice sheet models of the present type with regard to parameter variability is substantially increased if a region of high elevation is included. Since such plateaus are present in reality, there is not much reason to leave them out. In the present model high grounds are represented as shown in Figure 2. The dashed line shows the bedrock profile which is assumed to be in isostatic equilibrium if ice cover is absent.

The flow law employed here represents two modes of ice-mass discharge, namely, internal deformation and basal sliding. Given some stress, internal deformation becomes larger if the ice temperature (with respect to the pressure melting point) increases, e.g. Paterson (1969). In the present model no explicit calculation of deformation is done, so the total ice-mass discharge due to deformation should be directly related to ice temperature. Since most of the velocity shear is found in the lower layers of an ice sheet, it is natural to express $B$ in $\theta_{0}$, the basal ice temperature. Similarly, the effect of basal sliding on the total ice-mass discharge can be included by increasing $B$ according to the amount of melt water beneath the ice. In this study $B$ is parameterized as follows 


$$
B=4+6 e^{\theta_{0}^{\prime}}+\frac{2}{\pi} B_{\max } \arctan (W / 10) \mathrm{m}^{-3 / 2} \mathrm{yr}^{-1}
$$

Here, $\theta_{0}^{\prime}$ is the basal temperature with respect to the pressure melting point, $W$ is the amount of melt water beneath the ice and $B_{\max }$ is a constant that measures the effect of basal sliding on the ice-mass discharge.

According to an analysis by Weertman (1966), a large ice sheet frozen to the bottom will be roughly twice as thick as an ice sheet of similar size beneath which (sufficient) melt water is present. So extensive basal sliding is capable of halving the height-to-width ratio of a large ice sheet. In terms of the model described in this paper, if sufficient melt water is present $B$ should be such that this reduction of the ice thickness indeed occurs. Given the value of $B$ in the no-sliding case, the value of $B$ corresponding to the case with extensive sliding can be obtained by scaling (1) and (2). Another way is to compute a large steady-state ice sheet which is frozen to the bed, and then to vary $B$ until a steadystate ice sheet is produced with half the ice thickness of the former one. Both methods were carried out and appeared to give practically the same result. The value for $B_{\max }$ found in this way was about $120 \mathrm{~m}^{-3 / 2} \mathrm{yr}^{-1}$. However, the model behaviour for other values of $B_{\max }$ will also be discussed.

To compute the amount of basal water $\mathrm{W}$ and the flow constant $B$ it is necessary to know the temperature distribution within the ice sheet. This means that the thermodynamic equation for a continuum has to be solved with appropriate boundary conditions. In the present model this is done by an approximate method. It is assumed that the vertical temperature profile can be approximated by a second-order polynomial. The three coefficients appearing in this polynomial are thus a function of $x$ and $t$. Equations for these coefficients (the spectral equations) are obtained from the upper boundary condition (ice temperature equals atmospheric temperature), the lower boundary condition (the temperature gradient meets the geothermal heat flux) and the vertically-integrated form of the heat equation. The method is described in detail in the Appendix.

To close the system, an equation is needed that describes the budget of melt water beneath the ice. Unfortunately, very little is known about the processes that regulate the flow of melt water. Several objections can be made against the possibility of an extensive layer of water beneath the ice. Hydrostatic pressure exerted by the ice will probably tend to concentrate melt water at specific locations (to form 'lakes'). Lliboutry (1966) has stressed the importance of cavities filled with melt water. In principle, "channeling' may also occur, in particular in regions where bedrock slopes are large. In this rather hopeless situation, the best we can do probably is to employ an advection equation for melt water, i.e.

$$
\frac{\partial W}{\partial t}=-u^{\prime} \frac{\partial W}{\partial x}+S
$$

Here $S$ is the rate of melting at the base and $u^{\prime}$ is a fraction of the vertical mean horizontal ice velocity. In this study the fraction is taken 0.5 . The way in which melting or refreezing is calculated is described in the Appendix. 
The treatment of the thermal regime of an ice sheet and its effect on the ice flow as used in this study is admittedly crude and has many intuitive aspects. [Another approach to model glacier surges involving basal sliding has been carried out by Budd and McInnes (1975)]. Here one should remember the specific purpose of this study: to construct a model that can be used for integrations over one million years, but is nevertheless sophisticated enough to include the effects of bedrock sinking and thermodynamics in an essentially correct way.

Numerical integration of the model equations is performed on a grid with a spacing of $70 \mathrm{~km}$, with a simple forward-time central-space difference scheme. The time step varies from 10 to $1 \mathrm{yr}$, depending on how $B$ varies over the grid (a small step being used if large horizontal differences in $B$ occur). The advection terms are treated with the Lax-Wendroff scheme (e.g. Mesinger and Arakawa, 1976).

\section{Environmental Conditions}

Parameterization of the ice accumulation rate $M$ is very difficult and can hardly be done without ad hoc assumptions. In most approaches the concept of a snow line is used, see Figure 2. It separates the regions where $M>0$ from those where $M<0$. Weertman (1976) used constant values for $M$ in the ablation and accumulation regions while others (e.g. Andrews and Mahaffy, 1976) have employed a functional form for $M$ in terms of surface elevation with respect to the height of the snow line. Pollard (1980) includes a somewhat more explicit calculation of snow fall, but it is doubtful whether this yields essentially different results.

Here, we use a simple representation of $\mathrm{M}$, namely

$$
M=\min \left[M_{\text {up }}, 1.5 \times 10^{-3} \times(H+h-E)\right] m \text { ice } \mathrm{yr}^{-1} .
$$

$E$ is the snow line elevation, given by

$$
E=E_{0}+\alpha x,
$$

where $\alpha$ is the slope of the snow line and $E_{0}$ the height of the snow line at the northern tip of the continent. $M_{\text {up }}$ is an upper limit to the ice accumulation rate and should be imposed because for cold conditions the capability of the air to contain water vapour is very small (annual precipitation over the Arctic: about $0.4 \mathrm{~m} \mathrm{yr}^{-1}$; over the Antarctic continent: $0.17 \mathrm{~m} \mathrm{yr}^{-1}$ ). In studying the response of the model ice sheet to external forcing, $E_{0}$ will be varied. This simply means that the snow line moves up and down, while its slope and the vertical gradient in the ice accumulation rate are kept constant.

Other environmental conditions that have to be prescribed are connected with the heat balance of the ice sheet (see Appendix). The geothermal heat flux entering the base of the ice sheet is set to $0.04 \mathrm{~W} / \mathrm{m}^{2}$, which is slightly less than the global mean value (e.g. Paterson, 1980). The annual mean surface temperature $T_{a}$ is determined from

$$
T_{a}=T_{s}-0.01 \times(H+h-E) K,
$$


where $T_{s}$ is the 'snow-line temperature'. Data from the Greenland Ice Sheet (Putnins, 1976; Ambach, 1972) suggest that $T_{s}$ is in the -12 to $-15^{\circ} \mathrm{C}$ range. The atmospheric lapse rate along the surface used here is $0.01 \mathrm{~K} \mathrm{~km}^{-1}$. This figure is suggested by data from Antarctica and Greenland (note that it is larger than the lapse rate in the free atmosphere).

\section{Basic Model Behaviour}

In the discussion of this section thermodynamics are not yet taken into account. All properties of the model ice sheet discussed below apply to conditions with a constant flow parameter $B$.

The model ice sheet responds highly nonlinearly to changes in snow line elevation. The presence of a fixed ice boundary at $x=0$ creates branching of the steady-state solutions (Weertman, 1961). A typical solution diagram is shown in Figure 3a. It shows equilibria of the ice 'volume' $V$ as a function of the snow line elevation. If the snow line intersects the surface sufficiently far north from the coast $(x=0)$, no ice sheet is possible. If the intersection is on land, a large ice sheet must exist. In between is a region where both $V=0$ and $V=$ large are stable equilibria.

The presence of a mountain range causes another branching of the steady-state solutions (Oerlemans, 1981). A typical situation is shown in Figure 3b. For certain values of $E_{0}$, a small ice cap on the mountain is stable.

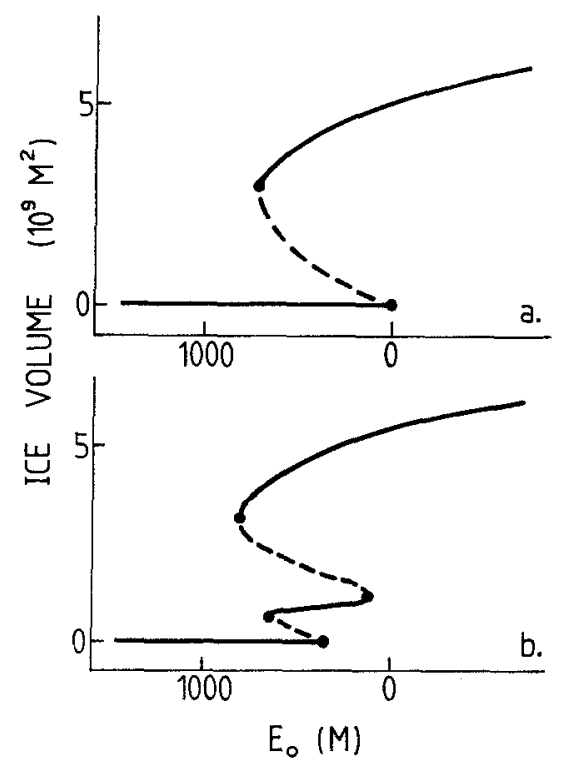

Fig. 3. Typical solution diagrams for a Northern Hemisphere ice sheet when there is no interaction between ice flow and ice temperature. Stable equilibria are indicated by solid lines, unstable equilibria by dashed lines. The upper panel refers to the case without a mountain range at high latitudes, the lower one to the case in which such a mountain range is present. Climatic conditions are reflected by $E_{0}$, the snow-line elevation at $73^{\circ} \mathrm{N}$ latitude. 
The location of the critical points depends on the model parameters, of course. A particularly important parameter is the slope of the snow line. For decreasing slope, the ' $E_{0}$-width' of the multiple equilibria region and the ice volume on the upper branche increase. However, for realistic model parameters, the structure of the system (without thermodynamics!) is always as shown in Figure 3.

To see how the model response to forcing depends on the slope of the snow line, we consider a few numerical experiments with periodic forcing. So $E_{0}=E_{0}^{*}+E_{\mathrm{am}} \cos (2 \pi P / t)$, where $E_{\mathrm{am}}$ is the forcing amplitude, $P$ the period and $E_{0}^{*}$ the mean snow-line elevation at $x=0$. Figure 4 summarizes the results of runs with $E_{\text {am }}=500 \mathrm{~m}$ and $P=22000 \mathrm{yr}$ (to mimic the effect of equinoxal precession on summer radiation). The upper curve shows the vertical movement of the snow line. In runs $a-d$ the flow parameter $B$ is constant and equal to $4 \mathrm{~m}^{-3 / 2} \mathrm{yr}^{-1}$. For a given slope $\alpha$ of the snow line, smaller values of $E_{0}^{*}$ lead to larger ice volumes. The transition from small mean ice volume to large mean ice volume appears to be more abrupt if $\alpha$ is smaller. Curves a and b correspond to $\alpha=0.8 \times 10^{-3}$ with $E_{0}^{*}=100$ and $0 \mathrm{~m}$, respectively. Curves $\mathrm{c}$ and d show results for $\alpha=0.5 \times 10^{-3}$ with $E_{0}^{*}=400$
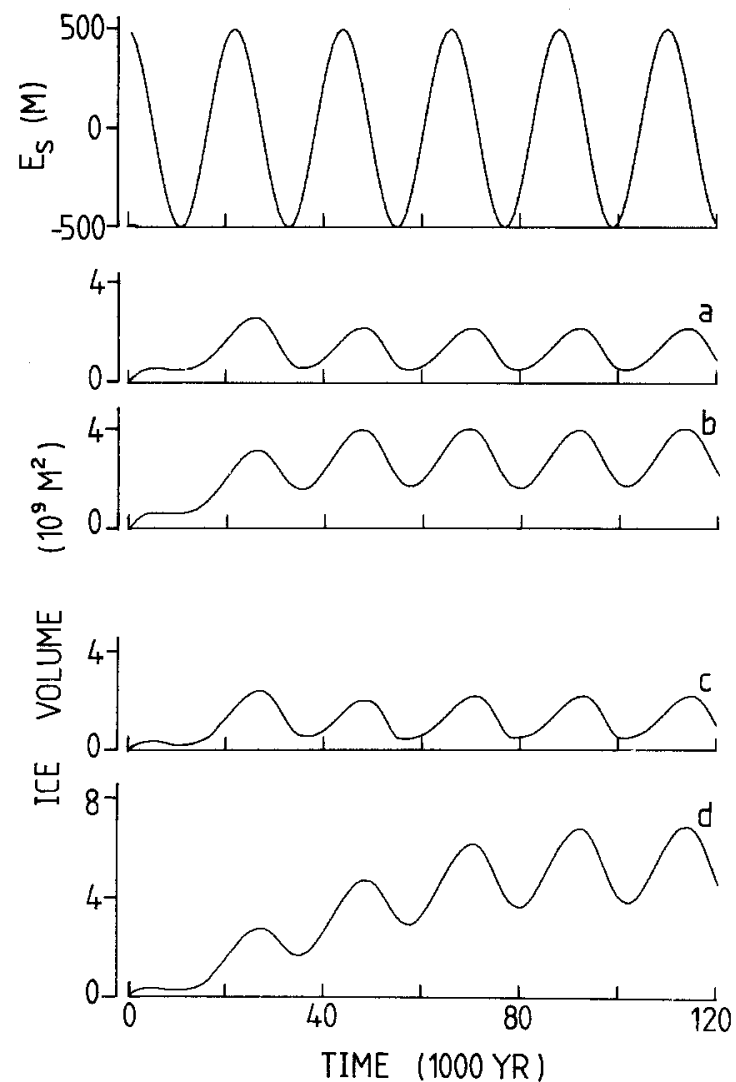

Fig. 4. Response of the model (no thermodynamics) to periodic forcing. The upper curve shows the snow-line elevation with respect to its mean value. Model parameters are: Curve $a: \alpha=0.8 \times 10^{-3}$, $E_{0}^{*}=100 \mathrm{~m}$; Curve b: $\alpha=0.8 \times 10^{-3}, E_{0}^{*}=0 \mathrm{~m}$; Curve c: $\alpha=0.5 \times 10^{-3}, E_{0}^{*}=400 \mathrm{~m}$; Curve d: $\alpha=0.5 \times 10^{-3}$, $E_{0}^{*}=300 \mathrm{~m}$. 
and $300 \mathrm{~m}$ respectively. Apparently, in the latter case the effect of a $100 \mathrm{~m}$ drop in $E_{0}^{*}$ is much larger.

According to these experiments, an ice sheet of ice-age size $\left(V \simeq 6 \times 10^{9} \mathrm{~m}^{2}\right)$ only forms if $\alpha$ is small. Large ice sheets can also be created for larger values of $\alpha$ if other parameters are adjusted, of course. Using a sufficiently small value of $E_{0}^{*}$ and a sufficiently large value of $E_{\text {am }}$ may lead to a large ice sheet even for $\alpha=10^{-3}$ (Weertman, 1976). However, present-day observations indicate that a value of $300 \mathrm{~m}$ for $E_{0}^{*}$ is already quite low. It also seems unlikely that the snow line moves up and down over a range much larger than $1000 \mathrm{~m}$, at least as long as changes in radiation associated with changes in the earth's orbit are concerned. In view of this, a value of $\alpha$ of about $0.5 \times 10^{-3}$ seems to be most suitable.

From Figure 4 we can also learn that the response of the model ice sheet to a 22000 yr forcing becomes essentially linear after some time. A signal in other periods than $22000 \mathrm{yr}$ is hardly present. This situation changes if the time scale for bedrock sinking $\left(t^{*}=5000 \mathrm{yr}\right.$ in this study) is substantially increased. For $t^{*}>12000 \mathrm{yr}$, which seems to be unrealistically large, a strong signal in the $80000-120000 \mathrm{yr}$ range appears (Oerlemans, 1980a).

The model behaviour sketched above becomes much more complicated if interaction between the temperature field and the ice flow is allowed. Before discussing experiments in which the complete model is forced, we first consider the possibility of free oscillations.

\section{Free Oscillations}

A number of experiments were carried out to see whether free oscillations are possible for a constant flow parameter $B$. Since bedrock adjustment lags ice volume, in principal free oscillations are possible. However, strong damping appeared to be present always, and no free oscillations were found.

This situation changes if thermodynamics are included. The strong positive feedback loop involving basal sliding (melting point is reached $\rightarrow$ increasing ice-mass discharge $\rightarrow$ increased frictional heating $\rightarrow$ more basal water $\rightarrow$ increasing ice-mass discharge, etc.) sets the stage for the occurrence of free oscillations. When a certain critical ice thickness is reached (depending on surface temperature, geothermal heat flux, ice accumulation rate, frictional heating), melt water is formed at the base of the ice sheet. In general, this happens near the thickest part of the ice sheet. Then the basal water spreads slowly towards the ice-sheet edge(s), and when the basal water reaches an edge, a surge takes place and the ice volume decreases rapidly. Now refreezing of basal melt water starts because the ice thickness has decreased substantially (the geothermal heat can escape 'easily' to the atmosphere). After some time all basal water has disappeared, and then the cycle starts again.

In Figure 5 a number of runs are shown in which free oscillations occur. The left-hand side shows ice volumes for a series of experiments in which only $E_{0}$ (snow-line elevation at $x=0$ ) is varied. Other model parameters are: $\alpha=0.5 \times 10^{-3}, B_{\max }=100 \mathrm{~m}^{-3 / 2} \mathrm{yr}^{-1}$, 

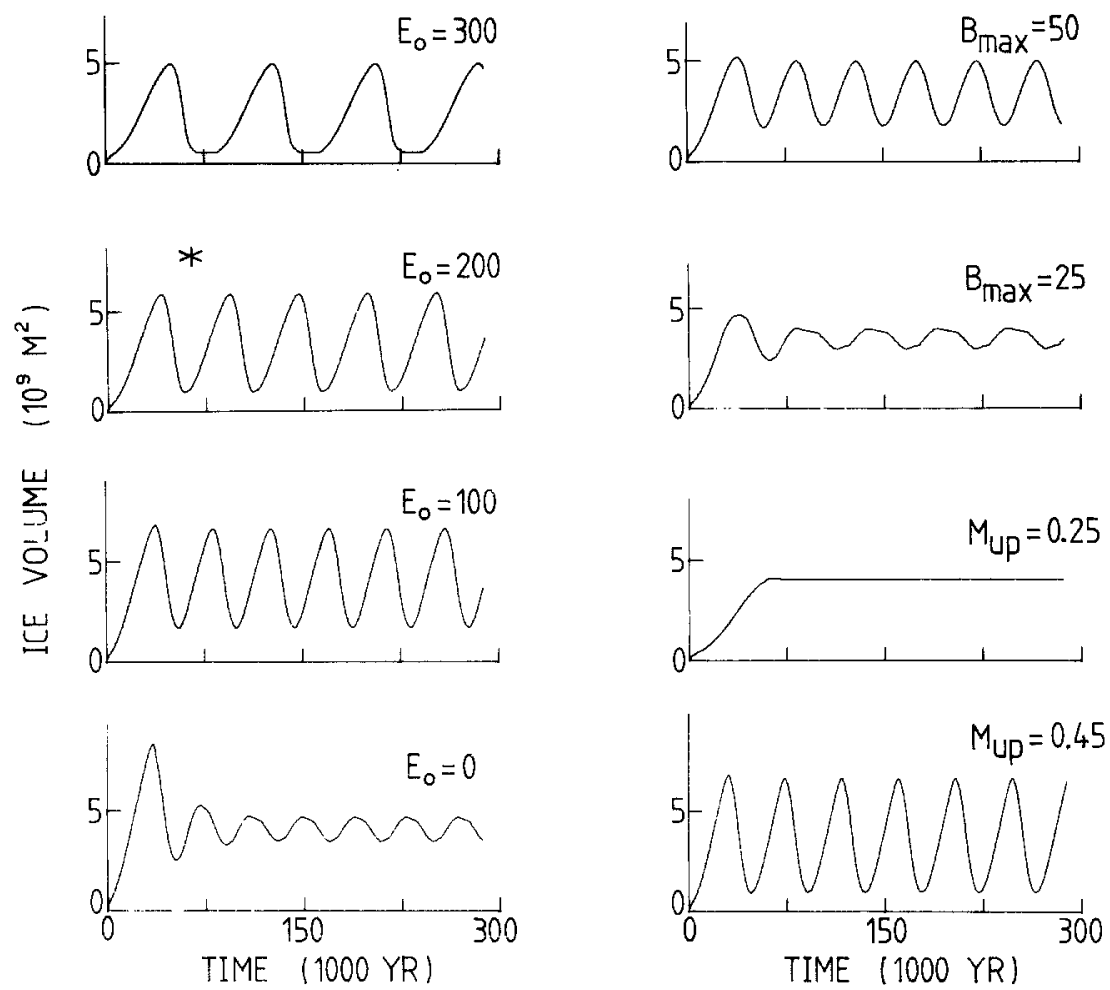

Fig. 5. Free oscillations occurring when thermodynamics are included. The left-hand side shows the effect of snow line elevation. Curves on the right-hand side show results from runs where the model parameters $B_{\max }$ and $M_{u} p$ were perturbed as indicated (with respect to model parameters used in the run marked with *).

$T_{s}=-14{ }^{\circ} \mathrm{C}$ and $M_{\mathrm{up}}=0.35 \mathrm{~m} \mathrm{yr}^{-1}$. Integrations were carried out over $300000 \mathrm{yr}$ of simulated time. For $E_{0}=400$, no oscillations occurred. For $E_{0}=300 \mathrm{~m}$, however, a strong cyclic signal is produced. It has a period of about $80000 \mathrm{yr}$ and ice volumes range over $5 \times 10^{9} \mathrm{~m}^{2}$. Note that the ice sheet never disappears completely: part of the mountain range is always above the snow line. Further lowering of the snow line leads to smaller periods. For $E_{0}=100 \mathrm{~m}$, the period is about $45000 \mathrm{yr}$.

If the snow-line elevation is decreased another $100 \mathrm{~m}$, the character of the solution changes. Now the surface temperature, which is coupled to the snow line, is so low that basal melting is restricted to the central part of the ice sheet. Melt water formed in the central part refreezes at the edges of the 'basal lake', and no large-scale surges are produced.

Figure 6 provides a closer look at the dynamics of one cycle. It corresponds to the first $70000 \mathrm{yr}$ of the second run in Figure $5\left(E_{0}=200 \mathrm{~m}\right)$. In this case, the production of basal water starts after $30000 \mathrm{yr}$, and reaches its largest values during the surge phase (which lasts comparatively short, but nevertheless takes thousands of years). Total refreezing of the bottom layer marks the start of a new cycle.

Free oscillations occur for a wide range of model parameters. As long as the snow line 


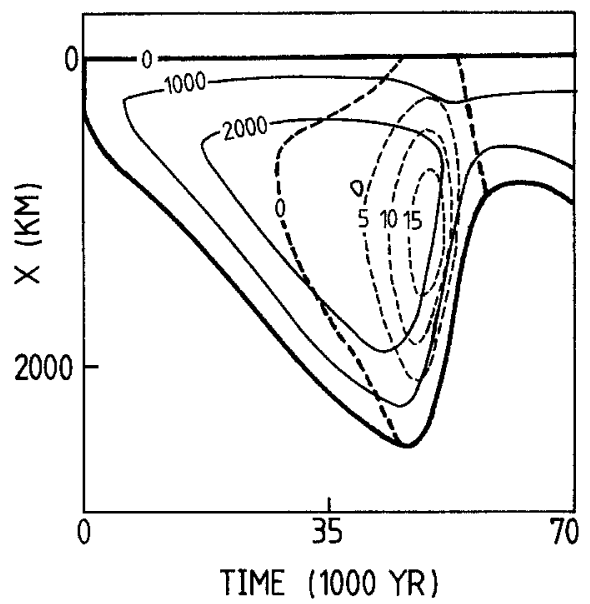

Fig. 6. Close-up of a free oscillation. Solid lines show ice thickness in $m$; dashed lines amount of basal water in $m$. The picture corresponds to the curve in the upper left of Figure 5.

is between certain limits (snow line too low: no basal melting; snow line too high: no large ice sheet), and $\left(\partial B / \partial \theta_{0}\right)_{\max }$ is above a critical value (which depends on other model constants), cyclic behavior is always encountered. The right-hand side of Figure 5 gives a few examples of model behaviour for other model parameters.

The run marked with * serves as control run. Perturbed parameters are indicated in the figure. Smaller values of $B_{\max }$ apparently lead to smaller amplitudes of the oscillation. For $B_{\max }=25 \mathrm{~m}^{-3 / 2} \mathrm{yr}^{-1}$, the oscillation is of a different nature. It is curious that a lower value of $B_{\max }$ results in smaller maximum ice volumes. The reason for this is rather subtle: it appears that a large value for $B_{\max }$, as used in the control run, leads to such a strong increase (if melt water occurs) of the southward ice-mass discharge that the ice thickness near the equilibrium point (snow line - ice surface intersection) increases. So at first instance the ablation zone shrinks and the ice volume continues to grow! The final surge, when the basal water reaches the ice-sheet edge, is more vigorous in case of large $B_{\max }$, of course.

The control run was also perturbed by changing $M_{\text {up }}$, the maximum ice-accumulation rate. For $M_{\text {up }}=0.25 \mathrm{~m} \mathrm{yr}^{-1}$, the ice sheet does not reach the size necessary for the formation of basal water. For $M_{\mathrm{up}}=0.45 \mathrm{~m} \mathrm{yr}^{-1}$, on the other hand, the cycle speeds up: the amplitude is larger now, and the period shorter $(\simeq 45000 \mathrm{yr})$.

It is obvious that in the present model free oscillations occur frequently. From the experiments it is also clear that model parameters can be chosen such that cycles with a period of $100000 \mathrm{yr}$ (and a sawtooth shape) are produced (the first run in Figure 5 comes close to it). In general, the period of the oscillation decreases with increasing $M_{\text {up }}$. Very long periods can be produced for small values of $M_{\mathrm{up}}$ and a very small slope of the snow line (otherwise, the ice sheet does not reach 'ice-age size'). 


\section{Response to Forcing}

The simplest way to force the model with the Milankovitch insolation variations is to set the snow-line elevation proportional to the caloric summer insolation at high latitudes. This yields the forcing shown in the top of Figure 7. Caloric summer insolation at $65^{\circ} \mathrm{N}$ was computed from expansions of the earth's orbital parameters given by Berger (1978) and from standard formulas to calculate insolation (e.g. Sellers, 1965).

All integrations shown in Figure 7 are started at $800000 \mathrm{yr}$ before present, with initial condition $h=H=0$. Runs $a, b$, and $c$ only differ in the value of $M_{u \mathrm{~b}}$; other model parameters for these runs are $E_{0}=300 \mathrm{~m}, B_{\max }=100 \mathrm{~m}^{-3 / 2} \mathrm{yr}^{-1}, T_{s}=-14^{\mathrm{b}} \mathrm{C}, \alpha=0.5 \times 10^{-3}$. Without any doubt, the simulated ice-volume curves are qualitatively similar to the oxygen isotope record shown in Figure 1. The response is quasi-periodic, and variations in ice volume

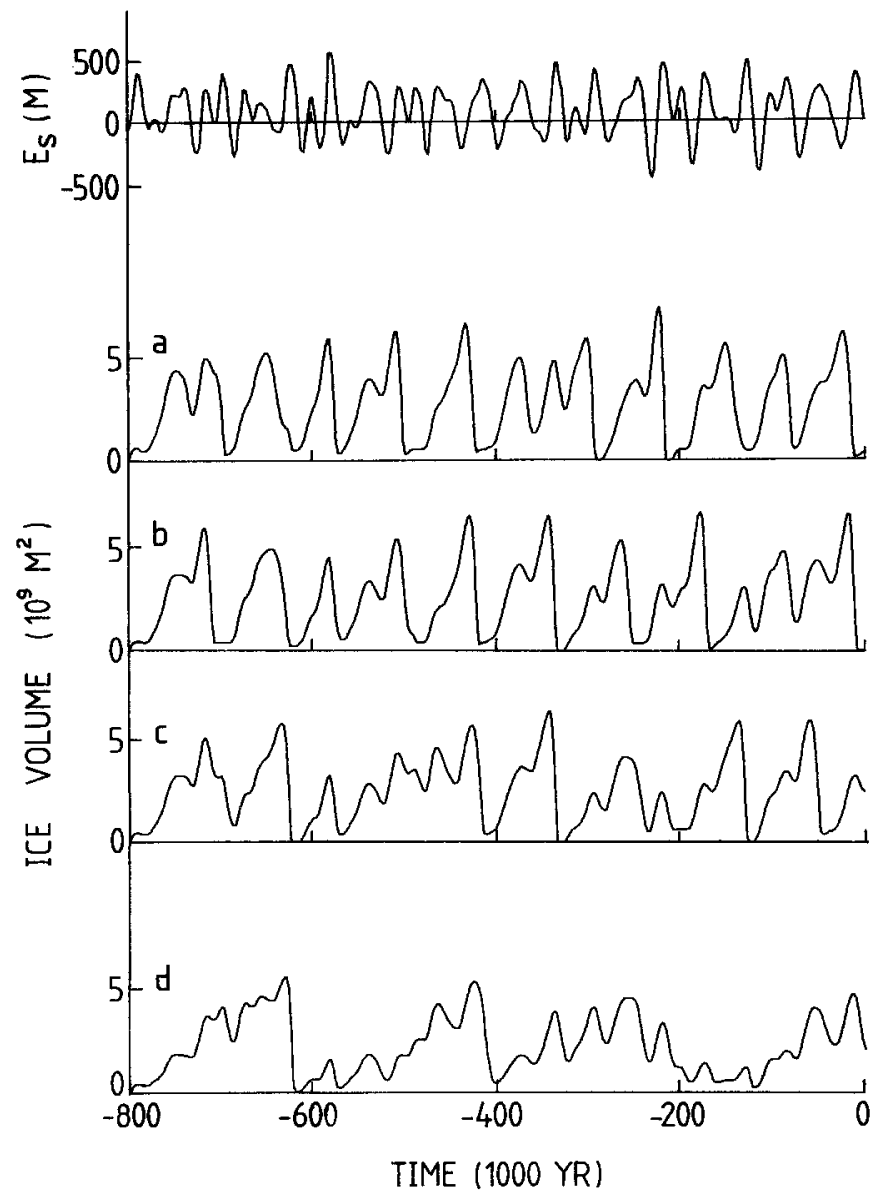

Fig. 7. Response of the model ice sheet to Milankovitch forcing. The upper curve shows the snow-line elevation with respect to its mean value. Curves a-c only differ in the value of $M_{\mathrm{up}}$, which is $0.4,0.375$, and $0.35 \mathrm{~m} \mathrm{yr}^{-1}$, respectively. The slope of the snow line for these runs is $0.5 \times 10^{-3}$. Curve $\mathrm{d}$ shows the result from a run with $M_{\mathrm{up}}=0.25 \mathrm{~m} \mathrm{yr}^{-1}$. In this case the slope of the snow line is $0.45 \times 10^{-3}$. 
are generally on a larger time scale than the radiation variations. Also, the sawtooth shape of the glacial cycles is well-reproduced. The runs show that the model response is very sensitive to the maximum ice accumulation rate. A similar sensitivity is encountered when other model parameters are varied. So the model response is of a stochastic periodic nature: glacial cycles with a sawtooth shape and a duration of 70000-130000 always show up, but there phase is not very stable with regard to parameter variability.

To illustrate that even longer cycles can be generated, curve $d$ in Figure 7 shows the result of a run in which $M_{\text {up }}=0.25 \mathrm{~m} \mathrm{yr}^{-1}$ andi $\alpha=0.4 \times 10^{-3}$. Other model parameters were not changed. In this case the model produces cycles with a duration of $200000 \mathrm{yr}$ !

Finally, two integrations were carried out in which the snow-line elevation was decreased gradually according to $E_{0}=500-t / 5000$, where $E_{0}$ is in $m$ and $t$ in yr. To be speculative, this could mimic a situation in which the northern hemisphere continents drift (strongly accelerated) in northward direction. Integrations were extended over $2000000 \mathrm{yr}$ of simulated time, with the model parameters of run $b$ in Figure 7 . The results are shown in Figure 8 . The upper curve shows a run without any additional forcing. Initially, a very small steady ice sheet is present over the mountains, but at a particular point the ice sheet starts to grow (a critical point in Figure 3 is passed) and free oscillations set in. The lower curve shows what happens when a white-noise forcing (standard deviation of snow-line elevation: $300 \mathrm{~m}$ for $2500 \mathrm{yr}$ periods) is added. In that case glacial cycles occur earlier, and the first cycles have a longer period (close to $100000 \mathrm{yr}$ ). So although environmental conditions change very slowly, the model ice sheet suddenly jumps into a glacial cycle regime of large amplitude. This feature is also found in many climatic records based on proxy data (e.g. Shackleton and Opdyke, 1973).

\section{Discussion}

The main conclusion of this study is that a continental ice-sheet model is capable of simulating the dominant characteristics of the global ice volume record. It appears that the dynamical interaction of ice thickness, bedrock sinking and heat accumulation in the

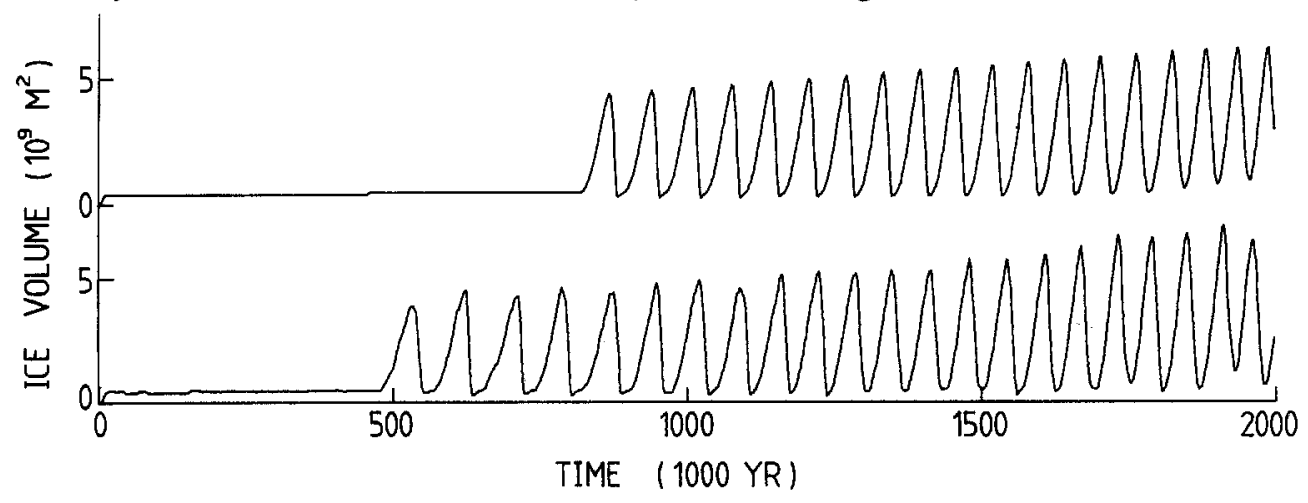

Fig. 8. Two long integrations in which the snow-line elevation decreases slowly (at a rate of $1 \mathrm{~m}$ per $5000 \mathrm{yr}$ ). The upper curve is for the case without additional forcing. The lower curve shows what happens if additional white-noise forcing is applied. 
ice sheet may create a more or less regular sequence of glacials and interglacials in which the famous sawtooth dominates. Although this is a promising result, a few reflections are useful.

First of all, model results are not very stable with respect to parameter variability. However, there is no reason to consider this as a model deficiency. It is very well possible that the pleistocene climate has a large stochastic component, and is almost unpredictable. This is the reason why it is difficult to match in detail the model simulation with the observed ice-volume record. The model can be tuned in such a way that the last two or three glacial cycles are well reproduced, but further back in time the right phase of the cycles is lost (Pollard, 1982 encountered a similar problem).

In general, tuning of the model is difficult because its behaviour is complex. This is due to the interaction of temperature field and ice flow. For example, an increase in $M_{\text {up }}$ makes the ice sheet thicker (so it is more difficult for the geothermal heat to escape), increases the frictional dissipation (because the total ice flow through the ice sheet is larger), but also increases the downward advection of cold ice. The net effect on the basal temperature may thus vary from place to place. When the model is forced, i.e. when the snow line moves up and down, it is not only the varying mass balance that affects the evolution of the ice sheet. An increased snow-line elevation implies higher temperatures at the ice surface. Such a temperature perturbation needs time to reach the base of the ice sheet. Optimal conditions for ice-sheet decay occur when a positive temperature perturbation (originating from a previous warm period) travels downward during a period of small snow-line elevation and reaches the base when the snow line goes up again. In this case large ice-mass discharge coincides with high melting rates. However, this mechanism is difficult to get hold on in tuning experiments, because the time necessary for the temperature perturbation to reach the base depends on ice thickness and accumulation rate.

Bedrock sinking plays an important role in rapid deglaciations, because it keeps a large part of the ice surface below the snow line once deglaciation has started. The time scale for bedrock sinking is by no means crucial (as it would be for an ice sheet that is never subject to melting, see Oerlemans, 1980a). The deglaciations occurring in the model would be even more rapid if a 'floating ice condition' would be built in, i.e. if ice that floats would not be considered anymore as belonging to the continental ice sheet. Due to the lagged bedrock sinking, the southern edge is mainly below sea level if the ice sheet shrinks. However, this is a passive mechanism that does not trigger deglaciation but merely accelerates the decay. Another situation arises when the rigidity of the lithosphere is taken into account. In that case the bedrock is also suppressed somewhat in front of the ice-sheet edge, and this may lead to high calving rates by invading water and initiate 'spontaneous' deglaciation. Pollard (1982) uses a scheme which yields this type of behaviour. Great care must be taken, however, if such processes are included in models with a fixed grid (in a simple scheme without special arrangements, a floating ice condition only permits retreat of the grounding line once the bedrock is below sea level; advance is not possible, see Oerlemans, 1982).

Still open to question is the role of the interaction between the Laurentide, Eurasian 
and Antarctic Ice Sheet. Budd (1981) has suggested that the $100000 \mathrm{yr}$ signal in the oxygen isotope record is completely due to variations in antarctic ice volume. It would be due to the fact that the Antarctic Ice Sheet needs about $70000 \mathrm{yr}$ to extend the grounded ice over the Ross and Weddell seas and to create conditions favourable for a major surge (initiated by rising sea level as a consequence of ice sheet decay in the Northern Hemisphere). Meanwhile, the Northern Hemisphere ice sheets would go through a number of full cycles (mostly three). Budd's suggestion is partly based on results of a model study of the Laurentide Ice Sheet (Budd and Smith, 1981). In this study an ice flow model was tuned in such a way that ice sheets of ice-age size were produced and disappeared again, without thermodynamic effects. This model did not generate $100000 \mathrm{yr}$ power, however. More detailed modeling of the Antarctic Ice Sheet is needed to see whether the antarctic ice volume reacts in such a way that it generates the $100000 \mathrm{yr}$ power in oxygen isotope spectra. In addition to this, it is unlikely that the evolution of the Laurentide and Eurasian ice sheets is always in phase. If the growth of one of them lags the other one in something like a regular sequence, a long time scale is added to the climate system. Before such effects can be modeled, we need to know much more about the factors that are decisive in the initiation of an ice sheet.

This brings us back to the uncertainty concerning the slope and mean elevation of the snow line. In short, one can state that variance of ice volume on larger time scales is produced when the slope of the snow line is smaller. In the case of small slope, a larger value of $E_{0}$ (which is much more in accordance with present-day conditions) can be used while the model still produces ice sheets of ice-age size. It appears that models using a steep snow line and, consequently, a small value for $E_{0}$, have difficulties in producing real interglacials (zero ice volume for some time). It therefore seems more likely that in the presence of growing ice sheets the slope of the snow line is smaller than present-day observations on mountain glaciers would suggest. It is not difficult to imagine that this might be the case. The ice sheet will exert a strong influence on its climatic environment, and some indication exists (Oerlemans and Vernekar, 1981) that accumulation over the southern part of a Northern Hemisphere ice sheet increases when it becomes larger (which effectively implies a reduction of the slope of the snow line).

In conclusion, this study has shown that a Northern Hemisphere ice-sheet model is capable of producing an ice-volume record that is similar in character to the record inferred from proxy data. Glacial cycles of long duration and asymmetric shape occur even without external forcing. The model results further indicate that the 'predictability' of global ice volume is rather low, due to the presence of nonlinear instabilities (in particular, basal sliding). If such an instability occurs, the model forgets the history of the ice sheet. Predictability should thus come from the direct model response to the Milankovitch forcing, which, according to this study, does certainly not explain the bulk of the variance in the global ice volume record.

\section{Acknowledgements}

The major part of the computations were carried out on the CRAY-1 of the European 
Centre for Medium Range Weather Forecasts (ECMWF). I like to thank ECMWF for the excellent working conditions provided during a number of visits. I also acknowledge useful comments from Cor Schuurmans, Dave Pollard and Michael Ghil.

\section{Appendix}

In this Appendix the thermodynamic part of the ice-sheet model is discussed and illustrated by a few simple model calculations.

The heat equation for a continuum reads

$$
\frac{\mathrm{d} \theta}{\mathrm{d} t}=k \nabla^{2} \theta+Q / \rho c
$$

Here, $\theta$ is temperature, $t$ is time, $k$ the thermal conductivity, $\rho$ density, $c$ specific heat and $Q$ the internal heat generated by velocity shear. A numerical solution in two-dimensional space is generally obtained by employing a two-dimensional grid. However, this is a (computer) time consuming procedure. Given the comparatively smooth temperature profiles observed in ice sheets and the fact that this paper deals with a very schematic ice-sheet model anyway, a more efficient approach is possible.

Expanding the ice temperature $\theta$ in powers of $h^{\prime}$, the height above the bedrock, and retaining three terms yields

$$
\theta\left(h^{\prime}, t\right)=\theta_{0}(t)+\theta_{1}(t) h^{\prime}+\theta_{2}(t) h^{\prime 2} .
$$

So it is assumed that the vertical temperature profile can be described by a second-order polynomial. Three equations are needed for $\theta_{1}, \theta_{2}$, and $\theta_{3}$ (spectral equations, in fact). These equations can be obtained from the lower and upper boundary condition, and from the vertically-integrated form of the heat equation.

As lower boundary condition the upward heat flux $G$ (the geothermal heat flux) at the base of the ice sheet $\left(h^{\prime}=0\right)$ is prescribed. This immediately yields

$$
\theta_{1}=-G / k \text {. }
$$

So if the geothermal heat flux is constant, $\theta_{1}$ is also constant. At the surface $\left(h^{\prime}=H\right)$ the ice temperature is set equal to the annual air temperature $T_{a}$. From Equations (A2) and (A3) it then follows that

$$
\theta_{2}=\left(T_{a}-\theta_{0}+G H / k\right) / H^{2} .
$$

To obtain the third equation, which should guarantee the conservation of heat, Equation (A1) is integrated from $h^{\prime}=0$ to $h^{\prime}=H$ :

$$
\begin{aligned}
\int_{0}^{H} \frac{\partial \theta}{\partial t} \mathrm{~d} h^{\prime}= & \left.k \frac{\partial \theta}{\partial h^{\prime}}\right|_{H}+G-\int_{0}^{H} w \frac{\partial \theta}{\partial h^{\prime}} \mathrm{d} h^{\prime}- \\
& -\int_{0}^{H} u\left(h^{\prime}\right) \frac{\partial \theta}{\partial x} \mathrm{~d} h^{\prime}+\int_{0}^{H} \frac{Q}{\rho c} \mathrm{~d} h^{\prime} .
\end{aligned}
$$


Diffusion in horizontal direction is omitted, because it is negligable compared to the advection of heat. At this point it is necessary to prescribe the profiles of $u$ and $w$, being the horizontal and vertical velocity respectively. For the present purpose it is sufficiently accurate to assume that the horizontal velocity profile is parabolic, i.e.

$$
u=\frac{3}{2} \mathrm{~h}^{\prime 1 / 2} / H^{3 / 2}\langle u\rangle,
$$

where $\langle u\rangle$ is the vertical mean horizontal velocity (as computed from the flow law, see section on model description). Since ice can be considered incompressible, it follows from the two-dimensional continuity equation that $w$ varies with $h^{3 / 2}$. Therefore it makes sense to write $w=w_{H}(h / H)^{3 / 2}$, where $w_{H}$ is the vertical velocity at the surface.

All terms in Equation (A5), except the internal generation of heat, can now be expressed in the coefficients $\theta_{i}(t)$. Eliminating $\theta_{1}$ and $\theta_{2}$ with Equations (A3) and (A4) yields, after considerable algebraic manipulation, an equation for the basal temperature $\theta_{0}$. It reads

$$
\begin{aligned}
\frac{2}{3} H \frac{\partial \theta_{0}}{\partial t}= & \theta_{0}\left(\frac{4}{7} W_{H}-2 k / H-\frac{2}{3} \frac{\partial H}{\partial t}\right)+\frac{\partial H}{\partial t}\left(\frac{2}{3} T_{a}+\frac{1}{3} G H / k\right)- \\
& -\frac{1}{3} H \frac{\partial T_{a}}{\partial t}+2 G+T_{a}\left(2 k / H-\frac{4}{7} W_{H}\right)-\frac{6}{35} W_{H} G H / k- \\
& -\langle u\rangle H \frac{\partial \theta_{0}}{\partial \mathrm{x}}-\frac{3}{7}\langle u\rangle H^{3} \frac{\partial}{\partial x}\left[\frac{1}{H^{2}}\left(T_{a}-\theta_{0}+G H / k\right)\right]+ \\
& +\int_{0}^{H} \frac{Q}{\rho c} \mathrm{~d} h^{\prime} .
\end{aligned}
$$

The surface vertical velocity is given by ( $h^{\prime}$ is positive upwards, so downward velocity is negative)

$$
w_{H}=-M+\frac{\partial H}{\partial t}+u_{H} \frac{\partial H}{\partial x}+S,
$$

where $u_{H}$ is the horizontal velocity at the surface, $M$ the ice accumulation rate at the surface and $\mathrm{S}$ the melting rate at the base (which is small compared to the other terms). It should be noted that $w_{H}$ is the vertical velocity relative to the vertical velocity due to a sloping bedrock (i.e. relative to $u_{H}(\partial h / \partial x)$. Also, in the derivation sketched above $u$ is in fact the velocity parallel to the local bedrock.

The last term in Equation (A7), the vertically-integrated frictional heating, can be computed from the release of potential energy in an ice column by downward motion. This is possible because the amount of kinetic energy associated with the ice flow is much smaller than the potential energy. It leads to

$$
\int_{0}^{H} \frac{Q}{\rho c} \mathrm{~d} h^{\prime}=-\frac{1}{\rho c} \int_{0}^{H} \rho g w_{*} \mathrm{~d} h^{\prime}=-\frac{\mathrm{g} H}{-c}\left(\frac{2}{5} W_{H}+\langle u\rangle \frac{\partial h}{\partial x}\right) .
$$


Now it is important to use the 'absolute' vertical velocity $w$ (in rigid motion of ice down a slope potential energy is released!). In practice, the $\langle u\rangle \partial h / \partial x$ term is smaller than the other term.

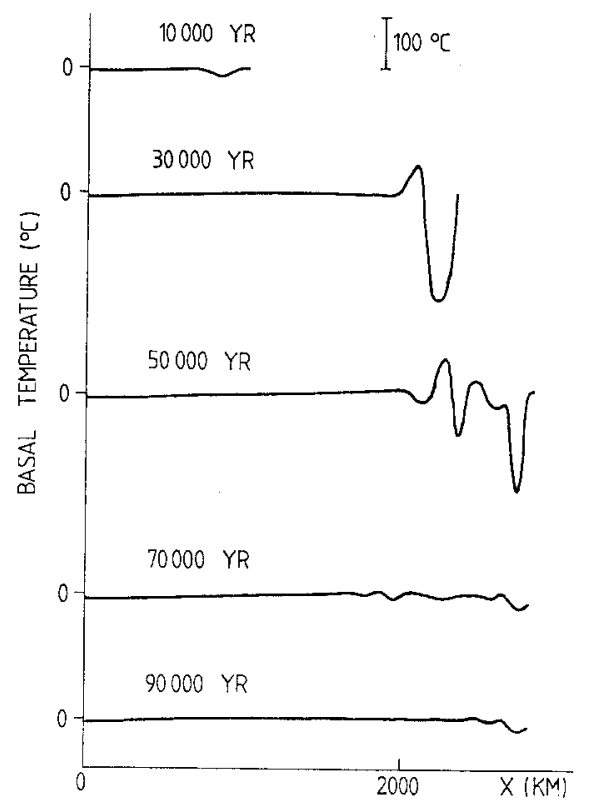

Fig. A1. The effect of truncation errors on the solution of the thermodynamic equation. The error is damped when the model ice sheet approaches a steady state.

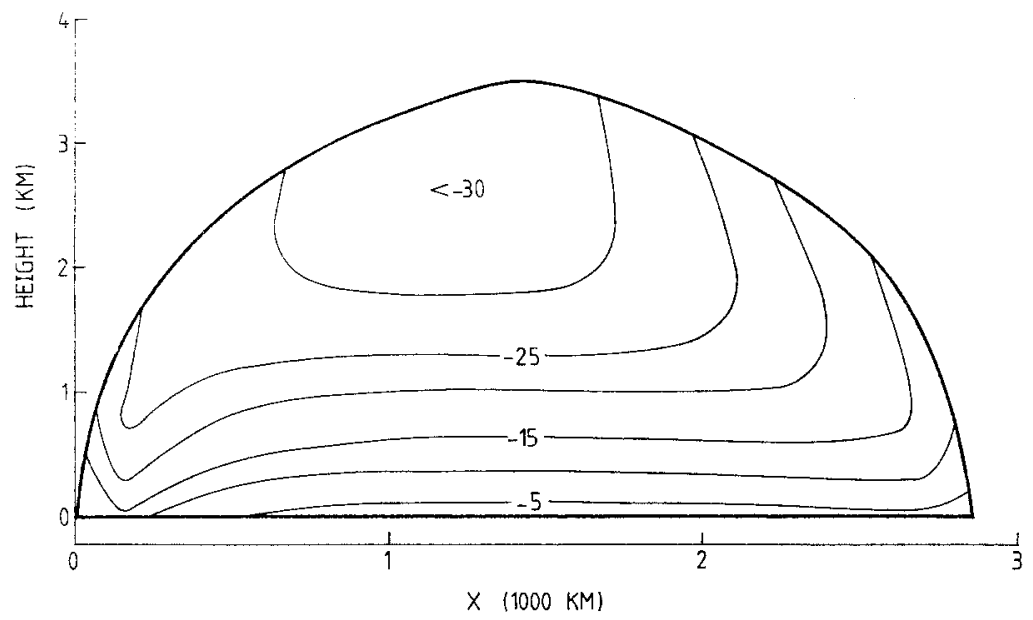

Fig. A2. A typical example of a temperature field calculated with the method described here. In this example, the model is run to a steady state for a fixed snow line (without bedrock adjustment). To prevent the occurrence of basal melting, a low value for the temperature at the snow line has been used. 
The rate of basal melting is computed by requiring that, as soon as $\theta_{0}$ equals the melting point (corrected for hydrostatic pressure), all surplus energy is used for melting. On the other hand, when basal water is present and the basal temperature tends to drop below the melting point, refreezing takes place. If sufficient melt water is present to permit basal sliding, part of the vertically-integrated frictional heating is used for additional melting of ice. This part varies between 0 and $50 \%$, and is a piecewise linear function of the flow constant $B: 0 \%$ for $B=B_{\min }$ and then increasing to $50 \%$ for $B=B_{\max }$.

No attempt was made to include a separate thermodynamic calculation in case of substantial sliding, in which case the velocity profiles are different. The reason for this is that also in the case without sliding most of the velocity shear is found in the lower layers. So in case of basal sliding horizontal velocities are larger (up to twice the regular 'frozen-to-the-bedrock' mean velocities), but all assumptions made in the foregoing discussion are retained.

The equation for $\theta_{0}$ is integrated on the same grid as used for the ice-flow model (see section on Model description). Several schemes for the integration in time were explored. It appeared that the Lax-Wendroff scheme (e.g. Mesinger and Arakawa, 1976) performed best. Although this scheme turned out to be numerically stable, some curious things did happen. This is illustrated in Figure A1, where $\theta_{0}$ is shown as a function of horizontal distance $x$ at various time. It concerns an ice sheet that grows southwards from $x=0$ (southern coast of the polar sea, say) to a steady state. The prescribed mass balance is constant. It is evident that large temperature fluctuations are created by the advancing snout of the ice sheet. If the sheet becomes steady, the fluctuations are damped.

A closer look at this problem revealed that these temperature fluctuations are caused by truncation errors. Assuming that the geothermal heat flux is constant ( $s o \partial \theta_{1} / \partial x=0$ ), the advection can be written

$$
\mathrm{ADV}=-\frac{3}{2}\langle u\rangle\left[\frac{\partial \theta_{0}}{\partial x}+\frac{3}{7} H^{2} \frac{\partial \theta_{2}}{\partial x}\right] .
$$

In order to meet the boundary conditions, $\theta_{2}$ becomes very large if the ice thickness is small (if the ice edge advances, ice thicknesses of a few meters appear in the model). In reality the second term within the square brackets does not vary too much near the edge because smaller values of $H^{2}$ are associated with larger values of $\partial \theta_{2} / \partial x$. However, in the model the truncation error in $\partial \theta_{2} / \partial x$ becomes so large that this compensation is not present. As a consequence, large differences in $H^{2} \partial \theta_{2} / \partial x$ appear between the grid points nearest to the edge.

To solve this problem, a maximum value of $15 \times 10^{-6}{ }^{\circ} \mathrm{C} / \mathrm{m}^{2}$ was set to $\theta_{2}$. With this condition included the scheme performed quite well. The conservation of heat is not seriously violated by this procedure, because larger values of $\theta_{2}$ only appear in one or two grid points where the ice thickness is very small.

Finally, a typical temperature distribution as calculated by the model described here is shown in Figure A2. The field is dominated by the vertical temperature gradient and tongues of cold ice pointing to the edges of the ice sheet. This is in full accordance with observations on present-day ice sheets (e.g. Paterson, 1980). 


\section{References}

Ambach, W.: 1972, 'Zur Schätzung der Eis-Nettoablation im Randgebiet des Grönlandischen Inlandeises', Polarforschung 42, 18-23.

Andrews, J. T. and Manhaffy, M. A. W.: 1976, 'Growth Rate of the Laurentide Ice Sheet and SeaLevel Lowering (With Emphasis on the 115000 BP Sea Level Low)', Quaternary Res. 6, 167-183.

Berger, A. L.: 1978, 'Long-Term Variations of Daily Insolation and Quaternary Climatic Changes', J. Atmos. Sci. 35, 2362-2367.

Birchfield, G. E.: 1977, 'A Study of the Stability of a Model Continental Ice Sheet Subject to Periodic Variations in Heat Input', J. Geophys. Res. 82, 4909-4913.

Birchfield, G. E. and Weertman, J.: 1978, 'A Note on the Spectral Response of a Model Continental Ice Sheet', J. Geophys. Res. 83, 4123-4125.

Birchfield, G. E., Weertman, J., and Lunde, A. T.: 1981, 'A Paleoclimatic Model of the Northern Hemisphere Ice Sheets', Quaternary Res. (in press).

Bodvarsson, G.: 1955, 'On the Flow of Ice Sheets and Glaciers', Jökull 5, 1 -8.

Budd, W. F.: 1981, 'The Importance of Ice Sheets in Long Term Changes of Sea Level and Climate', IAHS Pub. No. 131, 441-471.

Budd, W. F. and Smith, I. N.: 1981, 'The Growth and Retreat of Ice Sheets in Response to Orbital Radiation Changes', IAHS Pub. No. 131, 369-409.

Budd, J. F. and McInnes, B.: 1975, 'Modelling of Periodically Surging Glaciers', Science 186, 925-927.

Budyko, M. I.: 1969, 'The Effect of Solar Radiation Variations on the Climate of the Earth', Tellus 21, 611-619.

Coakley, J. A. and Wielicki, B.: 1979, 'Testing Energy Balance Climate Models', J. Atmos Sci. 36, $2031-2039$.

Dool, H. M, van den: 1980, 'On the Role of Cloud Amount on an Energy-Balance Model of the Earth's Climate', J. Atmos. Sci. 37, 939-946.

Gal-Chen, T. and Schneider, S. H.: 1976, 'Energy Balance Climate Modeling: Comparison of Radiative and Dynamic Feedback Mechanisms', Tellus 28, 108-121.

Ghil, M. and Le Treut, H.: 1981, 'A Climate Model with Cryodynamics and Geodynamics', J. Geophys. Res. $86,5262-5270$.

Hartmann, D. L. and Short, D. A.: 1979, 'On the Role of Zonal Asymmetries in Climatic Change', J. Atmos. Sci. 36, 519-528.

Hays, J. D., Imbrie, J., and Shackleton, N. G.: 1976, 'Variations in the Earth's Orbit: Pacemaker of the Ice Ages', Science 194, 1121-1132.

Howard, L. N.: 1979, 'Nonlinear Oscillations', in Hoppensteadt (ed.), Nonlinear Oscillations in Biology', Lectures in Applied Mathematics, Vol. 17, American Mathematical Society, 253 pp.

Imbrie, J., Donk, J. van, and Kipp, N. G.: 1973, 'Paleoclimatic Investigation of a Late Pleistocene Carribean Deep-Sea Core: Comparison of Isotopic and Faunal Methods', Quaternary Res. 3, 1038.

Jenssen, D.: 1977, 'A Three-Dimensional Ice Sheet Model', J. Glaciology 18, 373-390.

Källen, E., Crafoord, C., and Ghil, M.: 1979, 'Free Oscillations in a Climate Model with Ice-Sheet Dynamics', J. Atmos. Sci. 36, 2292-2303.

Lliboutry, L.: 1966, 'Bottom Temperatures and Basal Low-Velocity Layer in an Ice Sheet',J. Geophys. Res. 71, 2535-2543.

Lindzen, R. S. and Farrell, B.: 1977, 'Some Realistic Modifications of Simple Climate Models', $J$. Atmos. Sci. 34, 1487-1501.

Mesinger, F. and Arakawa, A.: 1976, 'Numerical Methods Used in Atmospheric Models', Vol. 1. GARP Publication Ser. No. 17,64 pp.

Nicolis, C. and Nicolis, G.: 1981, 'Stochastic Aspects of Climatic Transitions - Additive Fluctuations', Tellus 33, 225-234.

North, G. R. and Coakley, J. A.: 1979, 'Differences Between Seasonal and Mean Annual Energy Balance Model Calculations of Climate and Climate Sensitivity', J. Atmos. Sci. 36, 1189-1204. Oerlemans, J.: 1980a, 'Model Experiments on the 100 000-yr Glacial Cycle', Nature 287, 430-432. Oerlemans, J.: 1980b, 'On Zonal Asymmetry and Climate Sensitivity', Tellus 32, $489 \ldots 499$.

Oerlemans, J.: 1980 c, 'Continental Ice Sheets and the Planetary Radiation Budget', Quaternary Res. $14,349-359$. 
Oerlemans, J.: 1981, 'Some Basic Experiments with a Vertically-Integrated Ice Sheet Model', Tellus 33, 1-11.

Oerlemans, J.: 1982, 'Response of the Antarctic Ice Sheet to a Climatic Warming: A Model Study', J. Climatology 2, 1-11.

Oerlemans, J. and Vernekar, A. D.: 1981, 'A Model Study of the Relation Between Northern Hemisphere Glaciation and Precipitation Rates', Contr. Atm. Phys. 54, 352-361.

Paterson, W. S. B.: 1969, The Physics of Glaciers, Pergamon Press, 250 pp.

Paterson, W. S. B.: 1981, 'Ice Sheets and Ice Shelves', in Colbeck (ed.), Dynamics of Snow and Ice Masses, Academic Press, 468 pp.

Pollard, D.: 1978, 'An Investigation of the Astronomical Theory of the Ice Age Using a Simple ClimateIce Sheet Model', Nature 272, 233-235.

Pollard, D., Ingersoll, A. P., and Lockwood, J. G.: 1980, 'Response of a Zonal Climate-Ice Sheet Model to the Orbital Perturbations During the Quaternary Ice Ages', Tellus 32, 301-319.

Pollard, D.: 1982, 'A Simple Ice-Sheet Model Yields Realistic $100 \mathrm{k}$ yr Glacial Cycles', Nature 272, 233-235.

Putnins, P.: 1970, 'The Climate of Greenland', in Orvig (ed.), World Survey of Climatology, Elsevier, Vol. 14,128 pp.

Saltzman, B., Sutera, A., and Evenson, A.: 1981, 'Structural Stochastic Stability of a Simple AutoOscillatory Climatic Feedback System', J. Atmos. Sci. 38, 494-503.

Shackleton, N. G. and Opdyke, N. D.: 1973, 'Oxygen Isotope and Paleomagnetic Stratigraphy of Equatorial Pacific Core V28-238: Oxygen Isotope Temperatures and Ice Volumes on a $10^{5}$ and $10^{6}$ yr Scale', Quaternary Res. 3, 39-55.

Shackleton, N. G. and Opdyke, N. D.: 1976, 'Oxygen Isotope and Paleomagnetic Stratigraphy of Pacific Core v28-239, Late Pliocene to Latest Pleistocene', Geol. Soc. Am. Mem. 145, 449-464.

Schneider, S. H.: 1972, 'Cloudiness as a Global Feedback Mechanism: The Effects on the Radiation Balance and Surface Temperature of Variations in Cloudiness', J. Atmos. Sci. 29, 1413-1422.

Sellers, W. D.: 1969, 'A Global Climatic Model Based on the Energy Balance of the Earth-Atmosphere System', J. Applied. Meteor. 8, 392-400.

Sergin, V. Y.: 1979, 'Numerical Modelling of the Glaciers-Ocean-Atmosphere Global System', $J$. Geophys. Res. 84, 3191-3204.

Sutera, A.: 1981, 'On Stochastic Perturbation and Long-Term Climate Behaviour', Quart. J. Roy. Met. Soc. 107, 137-152.

Turcotte, D. L.: 1979, 'Flexure', Advances in Geophysics 21, 51-86.

Warren, S. G. and Schneider, S. H.: 1979, 'Seasonal Simulation as a Test for Uncertainties in Parameterizations of a Budyko-Sellers Zonal Climate Model', J. Atmos. Sci. 36, 1377-1391.

Weertman, J.: 1961, 'Stability of Ice-Age Ice Sheets', J. Geophys. Res. 66, 3783-3792.

Weertman, J.: 1966, 'Effect of a Basal Water Layer on the Dimensions of Ice Sheets', J. Glaciol. 6, 191-207.

Weertman, J.: 1976, 'Milankovitch Solar Radiation Variations and Ice Age Ice Sheet Sizes', Nature $261,17-20$. 